Mon. Not. R. Astron. Soc. 000,176(2012) Printed 9 June $2021 \quad$ (MN LATEX style file v2.2)

\title{
Accretion disc viscosity: what do warped discs tell us?
}

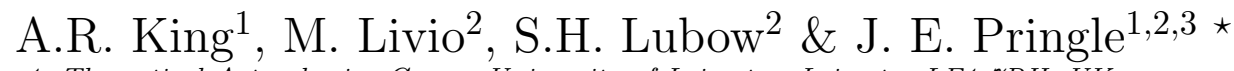 \\ 1. Theoretical Astrophysics Group, University of Leicester, Leicester LE1 7RH, UK \\ 2. Space Telescope Science Institute, 3700 San Martin Drive, Baltimore, MD 21218, USA \\ 3. Institute of Astronomy, University of Cambridge, Madingley Rd, Cambridge CB3 OHA, UK.
}

9 June 2021

\begin{abstract}
Standard, planar accretion discs operate through a dissipative mechanism, usually thought to be turbulent, and often modelled as a viscosity. This acts to take energy from the radial shear, enabling the flow of mass and angular momentum in the radial direction. In a previous paper we discussed observational evidence for the magnitude of this viscosity, and pointed out discrepancies between these values and those obtained in numerical simulations. In this paper we discuss the observational evidence for the magnitude of the dissipative effects which act in non-planar discs, both to transfer and to eliminate the non-planarity. Estimates based on the model by Ogilvie (1999), which assumes a small-scale, isotropic viscosity, give alignment timescales for fully ionized discs which are apparently too short by a factor of a few compared with observations, although we emphasise that more detailed computations as well as tighter observational contraints are required to verify this conclusion. For discs with low temperature and conductivity, we find that the timescales for disc alignment based on isotropic viscosity are too short by around two orders of magnitude. This large discrepancy suggests that our understanding of viscosity in quiescent discs is currently inadequate.
\end{abstract}

Key words: accretion, accretion discs

\section{INTRODUCTION}

Accretion discs have major importance for many branches of astrophysics. The fundamental process determining how they work is usually called viscosity. This determines the transport of mass and angular momentum within the disc, and as a result how much energy this releases. Despite considerable efforts, the physical origin of the viscosity is still at best only partially understood. This makes it worthwhile to consider what we can learn about it from the behaviour of observed disc systems. We attempt this here.

The simplest case of a plane disc has been extensively studied, so we review it only briefly before considering cases where the disc is tilted or warped, which is our main purpose in this paper. In this simplest case a geometrically thin Keplerian disc lies in a plane $(z=0$ in cylindrical polar coordinates $R, \phi, z)$ and the $R \phi$ stress transmitted by viscosity transports angular momentum outwards and mass inwards (Pringle 1981). The conservation laws for these two quantities combine to give an equation governing the radial motion of the surface density $\Sigma(R, t)$, which shows that the surface

* E-mail: jep@ast.cam.ac.uk density diffuses inwards on a timescale

$$
t_{\mathrm{visc}} \simeq \frac{R^{2}}{\nu}
$$

at disc radius $R$, where $\nu$ is the viscosity. For a disc semithickness $H \ll R$ we follow Shakura \& Sunyaev (1973) and define the dimensionless parameter $\alpha$ as a measure of the vertically averaged viscosity through the relation

$$
\nu=\alpha H c_{s} .
$$

Here $c_{s}$ is a vertically averaged measure of the disc sound speed, related to $H$ by hydrostatic equilibrium in the $z$ direction as

$$
c_{s}=H \Omega \text {. }
$$

Shakura \& Sunyaev (1973) comment that if the viscous mechanism is either hydrodynamic or magnetohydrodynamic turbulence, then rapid dissipation of such turbulence at supersonic or super-Alfvénic speeds is likely to ensure that $\alpha \leq 1$.

If the system is time-variable, as in dwarf novae or soft $\mathrm{X}$-ray transients, we get an estimate of $\nu$ or $\alpha$ by comparing the result (11) with the observed timescales of variability and estimates of the disc radius. For outbursts of both types 
of system, where the disc is fully ionized and gas pressure dominates, this suggests that $\alpha$ is in the range $0.1 \leq \alpha \leq 0.3$

(King, Pringle \& Livio, 2007; Kotko \& Lasota, 2012).

In the rest of this paper we ask what we can learn from observations of another aspect of disc viscosity, namely the $R z$ stress involved in possible tilting and warping of the disc.

\section{PROPAGATION OF DISC WARPS}

The response of gaseous discs to tilts or warps depends crucially on whether the viscosity is large or small, in the sense that it depends crucially on whether the radial communication of the tilt is mainly by pressure forces, or alternatively by viscous stress.

For high viscosity, i.e. $\alpha \gg H / R$, the warp is transmitted radially by viscous stresses. Here too, by consideration of conservation of mass and of angular momentum, for matter orbiting locally in a simple disc-like geometry, it is possible to obtain idealised equations for the evolution of the disc (both surface density and tilt), involving two viscous parameters: the usual $\nu$ relating to the $R \phi$-stress, and a second viscosity $\nu_{2}$ relating to the $R z$-stress (Papaloizou \& Pringle 1983, Pringle 1992). If we imagine each disc annulus to have a normal unit vector $\mathbf{l}(R, t)$, then for small disc tilt angle $\beta(R, t) \ll 1$, we have

$$
\mathbf{l}=(\beta \cos \gamma, \beta \sin \gamma, 0)
$$

in Cartesian coordinates, with $O Z$ perpendicular to the disc, and where $\gamma(R, t)$ represents the azimuth of the disc tilt at radius $R$ and time $t$. Then if we write $W \equiv \beta e^{i \gamma}$ (Petterson 1997a) and ignore external torques, for a steady disc (one for which $\Sigma$ is independent of time) the warp evolves as

$$
\frac{\partial W}{\partial t}=\frac{\nu_{2}}{2 R} \frac{\partial}{\partial R}\left(R \frac{\partial W}{\partial R}\right)
$$

So in these circumstances, warp propagation obeys a diffusion equation and the timescale on which the warp is smoothed out is approximately

$$
t_{\mathrm{damp}}=t_{\nu_{2}} \sim \frac{R^{2}}{\nu_{2}}
$$

Ogilvie $(1999,2000)$ has considered the particular case in which the disc viscosity can be assumed to be small-scale (i.e. mean free path much less than $H$ ) and isotropic, so that the disc fluid obeys the usual Navier-Stokes equations. He finds that the simplified equations given by Pringle (1992) provide an adequate description of the disc evolution, and there is an additional, lower order effect which gives rise to precession of the disc annuli. He also shows for small disc tilts (see also Lodato \& Price, 2010) that

$$
\frac{\nu_{2}}{\nu}=\frac{1}{2 \alpha^{2}} \frac{4\left(1+7 \alpha^{2}\right)}{4+\alpha^{2}} \text {. }
$$

For small values of $\alpha$ (but still with $\alpha>>H / R$ ) this gives

$$
\frac{\nu_{2}}{\nu} \approx \frac{1}{2 \alpha^{2}}
$$

This result is somewhat counter-intuitive, since it indicates that as $\alpha$ decreases, $\nu_{2}$ increases. The relationship comes about for Keplerian discs, for which the epicyclic frequency and orbital frequency almost coincide, so that a slight disc tilt produces a resonant response in internal horizontal disc motions (Papaloizou \& Pringle, 1983; see the discussion in Lodato \& Pringle 2007).

For the opposite case of low viscosity, that is $\alpha \ll H / R$, the warp is communicated radially by pressure. A small warp travels as a linear wave with wave speed $v_{w}=(1 / 2) c_{s}$, where $c_{s}$ is (an appropriate vertical average of) the local sound speed in the disc (Papaloizou \& Lin, 1985; Pringle, 1999). In this case the warp produces resonant horizontal shearing motions within the disc which are then subject to damping by the internal disc viscosity. Warp damping takes place over approximately $1 / \alpha$ orbital periods of the disc so that locally

$$
t_{\mathrm{damp}} \sim \frac{1}{\alpha \Omega} .
$$

\section{SYSTEMS WITH MISALIGNED DISCS}

In considering the evolution of misaligned discs, we first need to identify astrophysical systems in which the disc is thought to be misaligned. The most obvious candidates in this regard are semi-detached binary systems. In some of these the accretion disc around the mass-gaining star (here the primary) is for some reason misaligned with the binary orbit. It seems very likely that this misalignment results in some way from the presence of a binary companion orbiting outside the disc, although there is as yet no fully worked-out theory of this. In particular, although mass transfer from the companion may be an ingredient of the mechanism causing the misalignment, it cannot of itself be the direct cause: since mass transfer in the normal way supplies only an aligned component of angular momentum, it actually tends to reduce misalignment.

There are two main types of semi-detached binary system in which the accretion disc is thought to be misaligned - X-ray binaries which display long term 'superorbital' periods or modulations (summarised by Kotze \& Charles 2012), and cataclysmic variables displaying so-called 'negative superhumps' (summarised by Olech et al 2009). We discuss one example from each class, choosing the one which seems to give the most information about the viscosity in the system.

In each case the most readily observable quantity is the timescale on which the disc aligns with the orbital plane once the force driving the misalignment has been removed. To see what this tells us about the behaviour of viscosity in a warped disc we estimate the timescale for discs to align solely under the effect of viscosity. The realignment of the disc with the orbital plane then comes about through tidal torques which dominate at the outer disc edge. These tides cause differential precession of the outer disc elements. The resulting twist in the disc is then smoothed out by viscous effects, causing alignment. This viscous alignment timescale overestimates the true alignment timescale if significant mass transfer continues, since this adds aligned angular momentum to the disc, as noted above.

\subsection{Alignment timescale for tidally truncated discs}

If the disc radius is $R_{d}$, the orbital timescale is $\Omega=$ $\left(G M_{1} / R_{d}^{3}\right)^{1 / 2}$, where $M_{1}$ is the mass of the disc's central 
star (the primary). We write $1 / \omega_{p}$ as the timescale for (retrograde) precession induced by the tidal effect of the secondary star, so that the precession period is $P_{p}=2 \pi / \omega_{p}$.

\subsubsection{High viscosity, $\alpha>H / R$}

In this case, the warp is transmitted radially primarily by viscous stresses. Here we are concerned with what happens in the regime $t_{\nu_{2}} \omega_{p}<1$, when the viscous stresses are able to make the disc act as a cohesive whole (when this is not the case, differential precession is able to tear the disc into separate rings; Nixon et al. 2012).

For a steady disc subject to an external precessional torque the warp equation(5) can be written schematically as

$$
\frac{\partial W}{\partial t}=i \omega_{p} W+\frac{1}{2} \nu_{2} \frac{1}{R} \frac{\partial}{\partial R}\left(R \frac{\partial W}{\partial R}\right) .
$$

Multiplying this equation by $W^{\dagger}$, the complex conjugate of $W$, and then adding the resulting equation to its complex conjugate we get

$\frac{\partial}{\partial t}|W|^{2}=\frac{1}{2} \nu_{2}\left[\frac{W^{\dagger}}{R}\left\{\frac{\partial}{\partial R}\left(R \frac{\partial W}{\partial R}\right)\right\}+\frac{W}{R}\left\{\frac{\partial}{\partial R}\left(R \frac{\partial W^{\dagger}}{\partial R}\right)\right\}\right]$.

We take the integral of this over the disc, integrating the r.h.s. by parts and assuming zero torque boundary conditions. This gives

$$
\frac{\partial}{\partial t} \int|W|^{2} R d R=-\frac{1}{2} \nu_{2} \int\left|\frac{\partial W}{\partial R}\right|^{2} R d R .
$$

We can make estimates for each side of this equation. The l.h.s. is simply $\sim W^{2} / t_{\text {align }}$. To estimate the r.h.s., we note that if $t_{\nu_{2}} \sim 1 / \omega_{p}$ the viscous stresses acting to flatten the disc act on the same timescale as the precessional torques which tend to twist it up, and so we might expect that $\partial W / \partial R \sim W / R$. As the viscous timescale decreases, precession becomes less able to twist the disc, and so we can estimate for $t_{\nu_{2}} \omega_{p}<1$ that

$$
\frac{\partial W}{\partial R} \sim \frac{W}{R} \times\left(t_{\nu_{2}} \omega_{p}\right)
$$

Using these estimates, together with equations(2), (3) and (8) our estimate becomes

$$
t_{\text {align }} \approx \omega_{p}^{-1} \alpha^{-1}(H / R)^{2}\left(\Omega_{d} / \omega_{p}\right),
$$

where all quantities are evaluated at $R_{d}$.

\subsubsection{Low viscosity, $\alpha<H / R$}

The timescale for disc alignment in this case is considered by Bate et al. (2000) and by Lubow \& Ogilvie (2000). In the low viscosity limit the warp propagates as a linear wave at a speed $v_{w}=c_{s} / 2$. Provided that

$$
\omega_{p} \leq \frac{c_{s}}{R_{d}}
$$

the disc can precess as a whole (Papaloizou \& Terquem 1995, Larwood et al 1996); otherwise the disc can be torn apart (Larwood et al., 1996). This condition can also be written

$$
\frac{\omega_{p}}{\Omega_{d}} \leq \frac{H}{R}
$$

For the systems we are concerned with, Bate et al (2000) estimate that $\omega_{p} / \Omega_{d} \approx 0.01$ so that this inequality is easily satisfied. Then Bate et al. (2000) estimate the timescale for the disc to align with the binary orbit as

$$
t_{\text {align }} \approx \omega_{p}^{-1} \alpha^{-1}(H / R)^{2}\left(\Omega_{d} / \omega_{p}\right)
$$

(all quantities evaluated at $R_{d}$ ) which is exactly the same formula given in the high viscosity case.

\section{X-RAY BINARIES: HERCULES X-1/HZ HERCULIS}

The Hercules $\mathrm{X}-1 / \mathrm{HZ}$ Herculis system is a semi-detached binary system in which an $\mathrm{F}$ star, mass $M_{2} \approx 2 M_{\odot}$, transfers mass to a neutron star, mass $M_{1} \approx 1 M_{\odot}$. The binary period is $P=1.7 \mathrm{~d}$. This period is apparent both in $\mathrm{X}$-rays, because the neutron star undergoes eclipses by the $\mathrm{F}$ star, and in the optical, because the side of the $\mathrm{F}$ star facing the $\mathrm{X}$-ray source is so strongly heated that it appears more like an A star on that face. The system parameters are quite well determined because the radial velocity of the neutron star can be measured by observing the apparent change of its $1.24 \mathrm{~s}$ rotation period around the orbit. In addition, the $\mathrm{X}$-ray luminosity undergoes a regular variation with a period of $P_{\text {long }} \approx 35 \mathrm{~d}$. The $35 \mathrm{~d} \mathrm{X}$-ray flux curve for Her $\mathrm{X}-1$ consists of two peaks, equally separated in time, with a small residual flux in between. The larger peak lasts about 10 days, and the smaller peak, occurring about half a cycle later, lasts about 5 days (see the review by Priedhorsky \& Holt, 1987). Throughout the $35 \mathrm{~d}$ period the heating of the face of the companion star continues essentially unchanged, although there are subtle changes in the optical light curve. These changes (both the X-ray and the optical) led Gerend \& Boynton (1976) to propose a detailed model for the system in which the accretion disc around the neutron star is slightly tilted relative to the orbital plane and precesses retrogradely with a period of $35 \mathrm{~d}$. The model allows the secondary star to be visible to the $\mathrm{X}$-rays at all times during the $35 \mathrm{~d}$ cycle, but the illumination pattern on the face of the secondary varies with the synodic period $\left(1 / P+1 / P_{\text {long }}\right)^{-1}$ which is a few per cent less than the orbital period (for retrograde precession). Then, provided that the line of sight makes an angle to the orbital plane which is non-zero but slightly larger than the angle of disc tilt, the neutron star becomes directly visible (and so the $\mathrm{X}$-rays turn on) twice every precession period, with the larger and longer peak occurring when the disc is tilted towards the observer, and the smaller, shorter peak when the disc is tilted away.

The cause of the disc tilt is thought to be radiation warping (Petterson, 1977b,c; Pringle 1996). Modelling by Wijers \& Pringle 1999, and by Ogilvie \& Dubus 2001 shows that this can work provided that the mass input occurs at small radii (for example, Ogilvie \& Dubus assume that the stream adds mass at the circularization radius). This ensures that the disc shape is such that the radiation torques and the tidal torques at the disc edge act in the same retrograde direction. From their simulations Wijers \& Pringle find that the parameters of Her X-1 can be fitted, assuming that $\nu_{2} / \nu=1 / 2 \alpha^{2}$, with $\alpha=0.27$, as might be expected for a fully ionized disc (King et al 2007). Ogilvie \& Dubus take $\alpha=0.3$ and find that for this value Her $\mathrm{X}-1$ lies in that part 
of the parameter space where a steadily tilted and precessing disc is expected (see also Kotze \& Charles 2012). It is also worth remarking that, according to Ogilvie \& Dubus (2001), Her $\mathrm{X}-1$ lies close to the stability line, in that a reduction of the luminosity by a small factor would result in stability and an aligned disc.

So far this tells us little directly about $\nu_{2}$. But it does suggest that if the disc behaves as a Newtonian fluid (as in the theory of Ogilvie, 1999), the disc in Her $\mathrm{X}-1$ has $\alpha$ in the range $0.1 \leq \alpha \leq 0.3$. This is the same range needed to explain dwarf nova decay timescales for discs with similar physical properties. This range also implies that for the disc in Her $\mathrm{X}-1, \alpha \gg H / R \approx 0.04$ (Wijers \& Pringle, 1999) and so that warps propagate diffusively in this system, as we have assumed.

The most promising way of estimating $\nu_{2}$ is to find an observational measure of the rate at which disc realignment occurs when the forcing mechanism is turned off, or at least turned down. For a disc in which the warp is propagated diffusively, the realignment timescale is given by equation (14). If the observed precession is caused predominantly by tidal effects (cf. Larwood, 1998), we may assume that the dynamical driven precession timescale is comparable to the observed precession timescale $P_{\text {long }}$ (recall that the radiation torques which cause the disc tilt also provide disc precession at some level) so that $P_{p} \approx P_{\text {long }} \approx 35 \mathrm{~d}$. For the parameters of the Her $\mathrm{X}-1 / \mathrm{HZ}$ Her system, we take $q=M_{2} / M_{1}=2$, the ratio of disc radius to binary separation $R_{d} / a=0.24$, and the disc surface density as a power law $\Sigma \propto R^{-3 / 2}$, so that from Bate et al (2000) we obtain

$$
\frac{\omega_{p}}{\Omega_{d}} \approx 0.004
$$

Then using equation (14) and assuming that $\alpha \approx 0.3$, $H / R \approx 0.04$ and $\omega_{p} / \Omega_{p} \approx 0.004$, we find that

$$
t_{\text {align }}(\operatorname{HerX}-1) \sim 1.3 \omega_{\mathrm{p}}^{-1} \sim 7 \mathrm{~d} .
$$

From time to time the $\mathrm{X}$-ray flux of Her $\mathrm{X}-1$ drops and the system enters what is known as an anomalous low state. This occurred in 1983/84 (Parmar et al 1985), in 1998/99 (Coburn et al 2000; Still et al 2001) and in 2003/2004 (Jurua et al 2011, see also Kotze \& Charles 2012). Despite the Xray flux drop, the heating of the companion star HZ Her continues almost unchanged, indicating that although the disc inclination or disc shape obscures the direct line of sight to the neutron star, accretion continues and the face of much of the companion star is still able to receive $\mathrm{X}$-ray flux. As remarked above,the $35 \mathrm{~d} \mathrm{X}$-ray flux curve for Her X-1 consists of two peaks. The larger peak last about 10 days, and the smaller peak, occurring about half a cycle later lasts about 5 days. The tilted disc models suggest that during the large peak the disc is tilted towards the observer, and during the small peak it is tilted away from the observer (Gerend \& Boynton, 1976; Petterson, 1977c; Priedhorsky \& Holt, 1987). Thus an anomalous low state can come about if the disc becomes for some reason less inclined to the orbital plane, and thus more aligned with the line of sight (Coburn et al 2000).

Thus it seems reasonable to conclude that the onset of an anomalous low state is caused by the decrease in the disc inclination relative to the orbital plane, caused presumably by some decrease in the self-illumination of the disc and so a decrease in the physical cause of the warp. In this regard we note that Thomas et al (1983) remark that the amount of Xray heating of HZ Her seen in the period 1979 - 1982, prior to the 1983 anomalous low state, seemed to have decreased by around 15 per cent. The timescale on which this disc alignment takes place can be judged by the timescale for the onset of the anomalous low state. This appears (Coburn et al 2000, Still et al 2001) to occur on a timescale of order $P_{p}$.

In summary, although our estimated disc alignment timescale is slightly shorter than observations indicate, within the limits of the approximations made here, the whole picture fits reasonably well with a model in which warped disc motions are treated as those of a Newtonian fluid, subject to a Navier-Stokes viscosity, with $\alpha \approx 0.3$.

\section{DWARF NOVAE: V503 CYGNI}

The SU UMa subclass of dwarf novae have short orbital periods, in the range $1.3-2 \mathrm{~h}$, and display two types of outburst: normal outbursts and 'superoutbursts' (e.g. Warner, 1995). The latter occur less frequently, but are brighter and last longer. During the superoutbursts a photometric modulation, with period longer than the orbital period by a few per cent (dependent on mass ratio $q=M_{2} / M_{1}$ ), is some times apparent. This modulation is known as a 'superhump', and is thought to be caused by the disc becoming large enough to be driven eccentric by a 3:1 resonance (Lubow 1991a, 1991b), and precessing in a prograde direction. Some of these systems, of which a clear cut example we consider here is V503 Cyg (Harvey et al 1995), display a photometric modulation during superoutburst which has a period slightly shorter than the orbital period, again by a few percent. This would then correspond to a disc precessing in the retrograde direction and is known as a 'negative superhump'. The basic model for the origin of negative superhumps is outined by Wood and Burke (2007). They suggest that the disc is tilted with respect to the orbital plane, resulting in retrograde tidal precession, and that the modulation is caused by the accretion spot, where the mass transfer stream strikes the disc, varying in distance from the primary white dwarf, and thus varying in brightness. The question here is what causes the disc tilt, and under what circumstances such a tilt can be maintained.

For dwarf novae, the radiation warping discussed above fails by many order of magnitude, as indeed it does for many of the low mass X-ray binaries (Ogilvie \& Dubus 2001). In the absence of this, the major problem with any warping mechanism is that if the mass transfer stream remains in the orbital plane, so that the transferred matter has no component of angular momentum that lies in the orbital plane, the resultant disc must also lie in the orbital plane. What is required is a mechanism which imparts to the disc a net component of angular momentum that lies in the orbital plane. Currently the only viable mechanism proposed in the literature is the suggestion by Smak (2009) that the mass transfer stream through the inner Lagrange point $L_{1}$ has a component perpendicular to the disc plane which oscillates in phase with the binary period. He suggests that this comes about because the tilted disc enables the neighbourhood of the $L_{1}$ point to be heated in an asymmetric manner, and one 
which varies on the orbital period. It is worth remarking here that modulation of mass transfer caused by irradiation has been discussed by various authors (e.g. Arons, 1973; Basko \& Sunyaev, 1973; Viallet \& Hameury, 2007) and that a similar suggestion about diverting the mass transfer stream out of the orbital plane was made by Shakura et al (1999) in order to account for some of the dips seen in the $\mathrm{X}$-ray light curve of Her X-1.

This mechanism can only work when the illumination of the surface of the secondary is enough to influence the mass transfer process and so is likely to occur only during outburst and/or superoutburst. The preferential occurrence of negative superhumps in SU UMa systems (along with some novalikes) indicates that we may need superoutbursts where the mass transfer rate is higher, but that this might also occur because the secondaries in these systems have low mass and so low intrinsic surface brightness. Smak (2004: Fig. 1) plots the ratio of irradiation flux to intrinsic stellar flux at a point close to $L_{1}$ for a number of (un-named) dwarf novae. It is notable that in the systems with shorter periods, i.e. those which show superoutbursts, the ratio is much larger (in the range 150 - 200) compared to the longer period systems (all $<100$, and typically $<20$ ).

\subsection{Basic properties of V503 Cyg.}

The properties of V530 Cygni are discussed by Harvey et al (1995). The orbital period, derived from radial velocities, is $\mathrm{P}=111.9 \mathrm{~min}=0.078 \mathrm{~d}$. 1 The negative superhump has period $P_{-}=109.0 \mathrm{~min}$, which implies precession period of $P_{p}=4174 \min =2.90 \mathrm{~d}=37.2 \mathrm{P}$.

Dwarf nova outbursts are though to be caused by limit cycle behaviour, with the disc jumping between a state in which the disc is hot and fully ionized, and the viscosity and accretion rate are high, and a state which is cool, not fully ionized, and the viscosity and accretion rate are low (see e.g. Lasota, 2001). Models of dwarf nova outbursts (e.g. Cannizzo, 1994; Hameury et al 1998) typically have

outburst: $H / R \approx 0.02-0.03$ and $\alpha_{h}=0.1-0.3$.

quiescence: $H / R \approx 0.006$ and $\alpha_{c}=0.01-0.02$

Note that in both cases $\alpha>H / R$, and therefore we expect the warp to evolve viscously at all times. But we should also note that the limits on $\alpha$ in the low state are probably not well-defined. That is, although we do require $\alpha_{c} \ll \alpha_{h}$ in order to get outbursts of the right magnitude, it is possible that $\alpha_{c}$ could be a lot smaller than in these models. One method of trying to identify $\alpha_{c}$ is to model the variation of disc radius through the outburst cycle (cf. Ichikawa \& Osaki 1992). The smaller $\alpha_{c}$, the less the disc evolves in quiescence and so the smaller the quiescent disc. In this context we note the finding by Smak (1999) that discs in quiescence appear to be smaller than models predict and to lie well away from the outer disc tidal truncation radius. Therefore in the following analysis we consider $\alpha_{c}$ to be relatively ill-determined and see to what extent we can set independent limits.

1 Note that in many 'negative superhump' systems the orbital velocity has to be identified from among several photometric periods apparently present in the data.
The observations we are trying to account for are described by Harvey et al. (1995), and relate to the outburst behaviour of V503 Cyg in 1994. At that time the superoutbursts occurred every $88 \mathrm{~d}$, lasting $\approx 10 \mathrm{~d}$, and normal outbursts occurred every $30 \pm 3 \mathrm{~d}$, lasting $\approx 3 \mathrm{~d}$. Negative superhumps were seen throughout all stages of the eruption cycle (both in outburst and in quiescence) at approximately constant amplitude.

\subsection{Timescale estimates}

Suppose that the the masses in V503 Cyg take typical values of $M_{2}=0.2 M_{\odot}, M_{1}=0.8 M_{\odot}$, so that $q=0.25$ and the total mass $M=1 M_{\odot}$. Then with the binary period of $112 \mathrm{~min}$ we have a binary separation of

$$
a=5.34 \times 10^{10} \mathrm{~cm} .
$$

Taking for this mass ratio the mean radius of the Roche lobe to be $R_{L} \approx 0.5 a$, the tidal truncation radius, i.e. disc radius, is $R_{d} \approx 0.8 R_{L} \approx 0.4 a$. Using this, and the mass ratio $q=M_{2} / M_{1}=0.25$ we find (Bate et al., 2000) that for this system (again assuming a power-law surface density $\left.\Sigma \propto R^{-3 / 2}\right)$

$$
\frac{\omega_{p}}{\Omega_{d}} \sim 0.005
$$

Then with $\alpha_{h} \approx 0.2$ and $H / R \approx 0.03$ we find from equation(14) that during outburst the disc alignment timescale is

$$
t_{\text {align }}(\text { outburst }) \sim 0.92 \omega_{p}^{-1} \sim 0.42 \mathrm{~d} .
$$

Thus it looks as if maintaining the disc tilt through normal outbursts is problematic. We are therefore left with the necessity that the disc tilting mechanism operates also during ordinary outbursts in this system.

Thus in any case, we require the disc tilt to last a least throughout the $30 \mathrm{~d}$ period between ordinary outbursts, i.e. for 385 orbital periods, or 10 precession periods, and we also need to the disc tilt to be enhanced or at least maintained during normal outbursts.

The problem now is that during quiescence, in the standard models, $(H / R)^{2}$ decreases by a factor of $\sim 10-25$, which, although $\alpha$ decreases by of order $\sim 10$, implies that $t_{\text {align }}$ is just as small, or even smaller, in quiescence.

\subsection{Is $\alpha$ even lower in quiescence?}

This implies that we need to reduce $\alpha$ during quiescence below the value usually assumed for $\alpha_{c}$. But doing this has a significant consequence: it implies that we move into the regime where $\alpha<H / R$, and warp propagation occurs in a wavelike manner through pressure effects.

In this regime, the disc can precess as a solid body provided that (Bate et al 2000) $R_{d} / c_{s} \leq \Omega_{p}^{-1}$, or, provided that

$$
H / R>\omega_{p} / \Omega_{d} \approx 0.005,
$$

which is approximately satisfied.

Using $H / R \approx 0.006$ we find $t_{\text {align }} \sim 0.34(\alpha / 0.01)^{-1} \mathrm{~d}$. This implies that to get $t_{\text {align }} \geq 30 \mathrm{~d}$ we would require $\alpha \leq$ $10^{-4}$. 


\section{CONCLUSIONS}

\subsection{Her X-1/HZ Her}

The Her $\mathrm{X}-1 / \mathrm{HZ}$ Her system is roughly consistent with the simple Navier-Stokes viscosity approximation for modelling the evolution of disc tilt (Ogilvie 1999), provided that $\alpha \approx 0.3$ which is what is expected for a fully ionized disc, although the rough estimates indicate that the disc alignment timescale might be too small by a factor of a few. More accurate modelling is required before a definitive conclusion can be drawn.

This illustrates the importance of observing the behaviour of Her $\mathrm{X}-1$ during its anomalous low states, when the disc appears to align more closely with the orbital plane. Even more important will be to observe the system through a prolonged low state such as that reported by Jones, Forman \& Liller (1973), when mass transfer seems to have halted altogether in this system for a period of around 10 years.

\section{$6.2 \quad$ V503 Cyg}

In the dwarf nova V503 Cyg, to maintain the amplitude of the negative superhump throughout the outburst cycle that is from superoutburst to superoutburst, so for $\simeq 88 \mathrm{~d}$ - it is necessary that the mechanism which produces the disc tilt should be present during ordinary outbursts as well as superoutbursts. Otherwise the tilt is expected to decay through an ordinary outburst.

For the negative superhump to survive throughout the interoutburst period (of order 10 precession periods) requires that $\alpha \leq 10^{-4}$, a value much smaller than the value $\alpha_{c} \sim 0.01$ usually assumed during quiescence.

Indeed, $\alpha$ has to be sufficiently small that we are in the regime in which disc warp is propagated in a wavelike manner. For typical numbers, the wave crossing timescale for the disc is comparable to the precession period, so that the disc close to the limit at which is might be torn into segments.

\section{DISCUSSION}

Current thinking is that 'viscosity' results from MHD turbulence, using feedback via the magnetorotational instability (MRI). King et al. (2007) noted that for fully ionized discs, numerical modelling of this type does not appear to produce agreement with the observations. Most computational work involves shearing boxes, and seems to indicate that in the absence of a large-scale external magnetic field, the disc itself cannot provide a large enough viscosity (e.g. Bai \& Stone 2012) by at least an order of magnitude.

MRI-driven MHD turbulence derives its energy from radial angular momentum transfer, so it is unclear how relevant this is to the problem of warped discs considered here. As Pringle (1992) has remarked there is a radical difference in the reaction of an embedded magnetic field to a continuous $R \phi$ shear, compared to the oscillatory $R z$ shear which results from a disc warp. So it is important to ask how well models of disc behaviour based on Navier-Stokes viscosity (small mean free path, isotropic) manage to account for the evolution of disc warp.
We have seen that for fully ionized discs (such as that in Her X-1 and in dwarf novae in outburst) this theory seems to work adequately. We note that the initial rough estimates made here suggest that theoretical alignment timescales might be shorter than those observed by factors of a few. This could of course mean that the force causing the misalignment does not at first switch off completely, and slows the realignment beyond the viscous estimate. This seems unlikely for Her X-1, which is close to the stability boundary for radiation warping as we noted above. It is possible that some other mechanism, such as the effect of the neutron star magnetic field (e.g. Lai, 1999; Terquem \& Papaloizou, 2000; Murray et al., 2002) might maintain a residual tilt and slow the alignment, although this is unlikely for the outer disc. More detailed calculations are required before we can be sure of this discrepancy. This offers a challenge to MHD modelling: to what extent can this theory be made to agree with observations, both the $35 \mathrm{~d}$-period behaviour and the transition to anomalous low states. As King et al. (2007) emphasized, even to explain $\alpha \approx 0.3$ is likely to require taking the computations beyond shearing boxes towards more global simulations encompassing the whole disc (unless all discs have exactly the right amount of external magnetic field threading them). It is exactly such 3D global models which will be required to model the evolution of disc warps.

In quiescent dwarf nova discs the nature of the viscosity is unclear, since these discs are cool enough that the usual models of MRI and MHD turbulence are unlikely to apply (Gammie \& Menou, 1998). Using the Navier-Stokes viscosity model we have noted that although all models of dwarf nova outbursts seem to demand that $\alpha_{c} \sim 0.01$, the value of $\alpha$ required to allow negative superhumps to persist throughout the period between outbursts is smaller than this by at least two orders of magnitude. Thus either it is possible to produce models of DN outbursts with lower $\alpha_{c}$, or the Navier-Stokes model is inadequate.

This leaves us with two questions.

(i) does the 'viscosity' (whatever it is) act like a NavierStokes viscosity?

(ii) is it possible to develop numerical models that work, not just for fully ionized, strongly conducting discs, but also for low temperature, low conductivity discs?

Besides these two theoretical questions, it is clear that further observational work constraining the behaviour of warped and tilted discs would be very valuable.

\section{ACKNOWLEDGMENTS}

JEP thanks STScI for support from their Visitor Program. SHL thanks IoA, Cambridge, for support from their Visitor Programme.

\section{REFERENCES}

Arons, J., 1973, ApJ, 184, 539

Bai, X.-N., Stone, J.M., 2012, ApJ, in press (arXiv:1210.6661)

Bate, M.R., Bonnell, I.A., Clarke, C.J., Lubow, S.H., Ogilvie, G.I., Pringle, J.E., Tout, C.A., 2000, MNRAS 317, 773

Baslo, M.M., Sunyaev, R.A., 1973, Astr. Sp. Sci., 23, 117

Cannizzo, J.K., 1994, ApJ., 435, 389 
Coburn, W., Heindl, W.A., Wilms, J., Gruber, D.E., Satubert, R., Rothschild, R.E., Postnov, K.A., Shakura, N., Risse, P., Kreykenbohm, I., Pelling, M.R., 2000, ApJ, 543, 351

Gammie C. F., Menou K., 1998, ApJ, 492, L75

Gerend, D, Boynton, P.E., 1976, ApJ, 209, 562

Hameury, J.-M., Menou, K., Dubus, G., Lasota, J.-P., Huré, J.M., 1998, MNRAS, 298, 1048

Harvey, D., Skillman, D.R., Patterson, J., Ringwald, F.A., 1995, PASP, 107, 551

Ichikawa, S., Osaki, Y., 1992, PASJ, 44, 15

Jones, C., Forman, W., Liller, W., 1973, ApJ, 182, L108

Jurua, E., Charles, P.A., Still, M., Meintjes, P.J., 2011, MNRAS, 418,437

King, A.R., Pringle, J.E., Livio, M., 2007, MNRAS,

Kotko, I., Lasota, J.-P., 2012, A\&A, 545, A115

Kotze, M.M., Charles, P.A., 2012, MNRAS, 420, 1575

Lai D., 1999, ApJ, 524, 1030

Larwood, J., 1998, MNRAS, 299, L32

Larwood, J.D., Nelson, R.P., Papaloizou, J., Terquem, C., 1996, MNRAS, 282, 597

Lasota J.-P., 2001, NewAR, 45, 449

Lodato, G., Price, D., 2010, MNRAS, 405, 1212

Lodato, G., Pringle, J.E., 2007, MNRAS, 381, 1287

Lubow S. H., 1991a, ApJ, 381, 259

Lubow S. H., 1991b, ApJ, 381, 268

Lubow, S.H., Ogilvie, G.I., 2000, ApJ, 538, 326

Murray J. R., Chakrabarty D., Wynn G. A., Kramer L., 2002, MNRAS, 335, 247

Nixon, C., King, A., Price, D., Frank, J., 2012, ApJ, 757, L24

Olech, A., Rutkowski, A., Schwarzenberg-Czerny, 2009, MNRAS, 399,465

Ogilvie, G.I., Dubus, G., 2001, MNRAS, 320, 485

Papaloizou, J., Lin, D.N.C., 1985, ApJ, 438, 841

Paploizou, J.C.B., Pringle, J.E., 1983, MNRAS, 202, 1181

Papaloizou, J.C.B., Terquem, C., 1995, MNRAS, 274, 987

Parmar, A.N., Pietsch, W., McKechnie, S., White, N.E., Trümper, J., Voges, W., Barr, P., 1985, Nature, 313, 119

etterson, J.A., 1975, ApJ, 201, L61

etterson, J.A., 1977a, ApJ, 214, 550

Petterson, J.A., 1977b, ApJ, 216, 827

Petterson, J.A. 1977c, ApJ, 218, 783

Priedhorsky, W.C., Holt, S.S., 1987, Sp. Sci. Rev., 45, 291

Pringle, J.E., 1992, MNRAS, 258, 811

Pringle, J.E., 1996, MNRAS, 281, 357

Pringle, J.E., 1999, in 'Astrophysical Discs', ASP Conf. Ser. 160, 53

Shakura, N.I., Prokhorov, M.E., Postnov, K.A., Ketsaris, N.A., 1999, A\&A, 348, 917

Smak, J., 1999, ActA, 49, 431

Smak, J., 2004, ActA, 54, 181

Smak, J., 2009, ActA, 59, 419

Still, M., O'Brien, K., Horne, K., Hudson, D., Boroson, B., Vrtilek, S. Quaintrell, H., Fiedler, H., 2001, ApJ, 553, 776

Terquem C., Papaloizou J. C. B., 2000, A\&A, 360, 1031

Warner, B., Cataclysmic Variable Stars, Cambridge University Press, 1995

Wijers, R.A.M.J., Pringle, J.E., 1999, MNRAS, 308, 207

Wood, M A., Burke, C.J., 2007, ApJ, 661, 1042 